\title{
Real-World Experience of Adding Metformin in Pregnant Women with Type 1 Diabetes in a Chinese Population: A Retrospective Cohort
}

\author{
Fan Ping · Mingqun Deng · Xiao Zhai · Yingna Song • \\ Xinhua Xiao
}

Received: March 15, 2019 / Published online: April 26, 2019

(c) The Author(s) 2019

\begin{abstract}
Introduction: Glycemic control in pregnant women with type 1 diabetes (T1D) is challenging with only insulin, and the incidence rate of adverse perinatal outcomes is high. Increasing data have indicated the safety and effect of metformin in pregnancy; however, no relevant data are available in pregnant women with T1D. We aimed to investigate glycemic control and perinatal outcomes in pregnant women with T1D in a Chinese population and explored the role of metformin in these patients.
\end{abstract}

Fan Ping and Mingqun Deng contributed equally to this study.

Enhanced Digital Features To view enhanced digital features for this article go to https://doi.org/10.6084/ m9.figshare.7976489

F. Ping · M. Deng · X. Zhai · X. Xiao ( $₫)$

Key Laboratory of Endocrinology of National Health and Family Planning Commission, Department of Endocrinology, Peking Union Medical College Hospital, Chinese Academy of Medical Sciences, Peking Union Medical College, Beijing 100730, China

e-mail: xiaoxh2014@vip.163.com

Y. Song

Department of Gastroenterology, Peking Union

Medical College Hospital, Chinese Academy of

Medical Sciences, Peking Union Medical College,

Beijing 100730, China
Methods: We obtained data of 38 pregnant women with T1D who received regular antenatal care and delivered at Peking Union Medical College Hospital (PUMCH) between 1 January 2006 and 31 May 2018. The perinatal outcomes of T1D patients who added metformin as adjunct treatment and those who remained on insulin-alone therapy were compared retrospectively.

Results: Being overweight was common $(35.1 \%)$ in pregnant women with T1D. On average, the insulin dose increased by $35.30 \pm 22.60 \mathrm{unit} /$ day during pregnancy. The cesarean delivery rate was high $(65.8 \%)$, and fetal macrosomia was the main reason. The change of HbA1c in the metformin-insulin group was more prominent than in the insulinonly group $\quad(-1.47 \pm 1.17 \%$ vs. $\quad-0.90 \pm$ $1.13 \%, p=0.05)$. There was no statistically significant difference in perinatal outcomes between the two groups.

Conclusions: The incidence of adverse perinatal outcomes in pregnant women with T1D was high. This study innovatively suggested that metformin could be safe and could contribute to improving glucose management in pregnant women with T1D.

Keywords: Metformin; Perinatal outcomes; Pregnancy; Type 1 diabetes 


\section{INTRODUCTION}

Pre-gestational diabetes (PGDM) is diabetes diagnosed before pregnancy, including type 1 diabetes (T1D), type 2 diabetes (T2D) or other special types of diabetes. Compared with gestational diabetes mellitus (GDM), patients with PGDM are at higher risk of adverse perinatal outcomes, which include maternal preeclampsia, babies that are large for gestational age (LGA) or have macrosomia, perinatal death, neonatal congenital malformation, neonatal hypoglycemia and hyperbilirubinemia $[1,2]$. Among those with PGDM, the risk of poor perinatal outcomes in pregnancy complicated by T1D is higher than that for T2D [3]. A study from Sweden found that pregnant women with T1D had an eightfold risk of macrosomia compared with normal pregnant women [4]. However, the rate of pregnant women with T1D achieving target glycated hemoglobin (HbA1c) levels is low. In the UK, despite intensive support, only $15 \%$ of pregnant women with T1D achieve the target glycated hemoglobin (HbA1c) levels during early pregnancy, and only $40 \%$ achieve the target HbA1c levels after 24 weeks' gestation [5]. Improvement of glycemic control in pregnant women with T1D is urgently required. The incidence rate of T1D is relatively low in China, and there are few studies focused on pregnant women with T1D in the Chinese population.

It is well known that insulin resistance is prominent during pregnancy. The insulin dose can increase dramatically from pre-pregnancy to the third trimester $(0.618-1.060$ unit $/ \mathrm{kg})$ in T1D patients [6]. However, an increased insulin dose results in more maternal weight gain, which also contributes to poor perinatal outcomes [7]. In non-pregnant T1D patients, metformin significantly improved insulin sensitivity, thus reducing the insulin dose [8]. So far, there have been no reports of metformin in pregnant women with T1D, although there have been many studies on GDM or pregnant women with T2D suggesting its safety and effect [9].

Therefore, we aimed at investigating glycemic control and perinatal outcomes in pregnant women with T1D in a Chinese population and exploring whether metformin has a role in these patients. In this study, we retrospectively analyzed the clinical data of pregnant women with T1D in Peking Union Medical College Hospital (PUMCH) in terms of blood glucose management and perinatal outcomes. Furthermore, we compared patients who added metformin as adjunct treatment with those on insulin alone.

\section{METHODS}

We included T1D patients with singleton pregnancy who attended prenatal care and delivered in PUMCH between the 1 January 2006 and 31 May 2018. TID was defined as patients having any one of the following criteria: (1) one or more positive islet autoantibodies; (2) insulin use only on the diagnosis date and a non-insulin glucose-lowering drug, if any, was introduced 6 months later. Pregnant women with T1D who had ever taken any dose of metformin during pregnancy were grouped as metformin plus insulin. T2D, GDM and special types of diabetes were all excluded. The study was approved by the Ethics Committee of PUMCH (approval no. ZS-1380), and the patients' written informed consents were obtained. The study was performed in accordance with the Declaration of Helsinki.

Electronic and handwritten medical records were all reviewed, and telephone visits were made when needed information was missing from medical records. Age, duration of diabetes, body mass index (BMI) and insulin injection methods were recorded. Weight, glycated hemoglobin (HbA1c) and insulin dose requirement were compared between pre-pregnancy and the third trimester. Pre-pregnancy HbA1c was defined as the last HbA1c tested within 3 months before pregnancy. HbA1c per trimester was defined as a single or a mean level during that trimester. Insulin dose recorded at the last visit within 3 months before pregnancy was defined as the pre-pregnancy insulin dose. Insulin dose recorded at the last visit at the obstetrics clinic before delivery was defined as insulin dose in the third trimester. Perinatal outcomes were recorded, including gestational weeks of delivery, 
modes of delivery, preeclampsia or eclampsia, maternal weight gain, polyhydramnios, premature rupture of membranes, laceration of the perineum or lateral episiotomy, weight retention after 6 weeks of delivery, 1-min Apgar Score, macrosomia or LGA, low birth weight (LBW) or small for gestational age (SGA), neonatal hypoglycemia, neonatal intensive care units (NICU) admission, shoulder dystocia, neonatal jaundice and congenital malformations. Perinatal outcomes were also compared between patients in the metformin-insulin group and the insulinonly group. Overweight was defined as prepregnant $\mathrm{BMI} \geq 24 \mathrm{~kg} / \mathrm{m}^{2}$. Macrosomia was defined as birth weight of $>4000$ g; LGA was defined as a weight above the 90th percentile for that gestational age of Chinese infants. LBW was defined by the World Health Organization as a infant birth weight $\leq 2499 \mathrm{~g}$, regardless of gestational age. SGA newborns were those who were smaller than normal for the gestational age, defined as a weight below the 10th percentile for the gestational age of Chinese infants. Neonatal hypoglycemia was defined as $<2.2 \mathrm{mmol} / \mathrm{l}$ within $24 \mathrm{~h}$ and $<2.8 \mathrm{mmol} / \mathrm{l}$ after $24 \mathrm{~h}$, and neonatal jaundice was defined as hospitalization for neonatal hyperbilirubinemia.

\section{Statistical analysis}

Statistical analysis was performed using SPSS 24.0 (SPSS, Inc). Descriptive analysis results were expressed as mean \pm standard deviation (SD) and percentages (\%). The chi-square test or Fisher's exact test, where appropriate, was used for categorical variables. For normally distributed continuous variables, Student's $t$ test was used. The Mann-Whitney $U$ test was used for nonparametric variables. One-way ANOVA was also used when the change of HbA1c was analyzed. $p<0.05$ was accepted as a statistically significant difference.

\section{RESULTS}

Forty-one patients with T1D delivered in our hospital from 1 January 2006 to 31 May 2018. One patient complicated by severe thrombocytopenia was excluded. Another two patients were excluded because they did not receive antenatal care in our hospital. Thirty-eight pregnant women with T1D (mean age $30.39 \pm 3.48$ years, BMI $22.35 \pm 2.96 \mathrm{~kg} / \mathrm{m}^{2}$ ) were retrospectively analyzed. The mean duration of diabetes was $7.91 \pm 5.80$ years; $35.1 \%$ of patients were overweight. The mean HbA1c before pregnancy was $7.16 \pm 1.32 \%$. The prepregnancy insulin dose was $0.64 \pm 0.23$ unit/ kg day on average. HbA1c decreased by $1.10 \pm 1.15 \%$ on average from pre-pregnancy to the third trimester, and the mean $\mathrm{HbA1c}$ was $5.98 \pm 0.82 \%$ in the third trimester. The average amount of insulin increased $35.30 \pm 22.60$ unit/day during pregnancy, and the mean amount of insulin in the third trimester was $1.11 \pm 0.44 \mathrm{unit} / \mathrm{kg}$ day (Table 1 ). The cesarean delivery rate was as high as $65.8 \%$. Indications for cesarean section included macrosomia, malposition, contracted pelvic outlet, history of previous cesarean section and poor glucose control. For vaginal deliveries, the rate of laceration of the perineum or lateral episiotomy was high $(76.9 \%)$. Weight retention at 6 weeks postpartum was common $(87.1 \%)$, with an average retention weight of $4.85 \pm 4.07 \mathrm{~kg}$. The mean birth weight was $3513.95 \pm 806.37 \mathrm{~g}$, including three cases of LBW/SGA and 16 cases of macrosomia/LGA, which was the most common neonatal complication (42.1\%). Although there was no difference in pre-pregnancy BMI between mothers of babies with macrosomia/ LGA and normal gestational age (NGA) $(p=0.713)$, gestational weight gain in mothers of macrosomia/LGA babies was higher than for NGA $(p=0.033)$. Neonatal hypoglycemia was the second most common neonatal complication $(28.9 \%)$, and three newborns were admitted into the NICU because of hypoglycemia. In addition, there were $4 / 38$ cases of neonatal malformations. Two neonatal malformations occurred in the metformin-insulin group: one with polydactyly and the other with a slightly separated pelvis. Mothers of the two newborns had been prescribed metformin in pre-pregnancy and early-pregnancy, respectively. Levels of $\mathrm{HbA1c}$ in pre-pregnancy were high in both mothers, with $8.1 \%$ and $7.0 \%$, respectively. There were also two neonatal malformations in the insulin-only group. One was polydactyly 
Table 1 Characteristics of T1D patients and comparison of groups

\begin{tabular}{|c|c|c|c|c|}
\hline Variables & & Metformin-insulin & Insulin only & $p$ value \\
\hline Age (years) & $30.39 \pm 3.48(n=38)$ & $30.30 \pm 4.99(n=10)$ & $30.43 \pm 2.89(n=28)$ & 0.828 \\
\hline Duration (years) & $7.91 \pm 5.80(n=38)$ & $4.83 \pm 4.33(n=10)$ & $9.01 \pm 5.93(n=28)$ & 0.055 \\
\hline BMI $\left(\mathrm{kg} / \mathrm{m}^{2}\right)$ & $22.35 \pm 2.96(n=37)$ & $22.21 \pm 3.22(n=10)$ & $22.40 \pm 2.91(n=27)$ & 0.824 \\
\hline Overweight & $35.1 \%(n=37)$ & $30.0 \%(n=10)$ & $37.0 \%(n=27)$ & 1.000 \\
\hline Chronic complications of diabetes & $7.9 \%(n=38)$ & $0.0 \%(n=10)$ & $10.7 \%(n=28)$ & 0.552 \\
\hline CS-II & $34.2 \%(n=38)$ & $30.0 \%(n=10)$ & $35.7 \%(n=28)$ & 1.000 \\
\hline First parity & $92.1 \%(n=38)$ & $80.0 \%(n=10)$ & $96.4 \%(n=28)$ & 0.164 \\
\hline Pre-pregnancy HbAlc (\%) & $7.16 \pm 1.32(n=30)$ & $7.65 \pm 1.24(n=10)$ & $6.92 \pm 1.32(n=20)$ & 0.033 \\
\hline Late-pregnancy HbAlc (\%) & $5.98 \pm 0.82(n=28)$ & $6.09 \pm 1.05(n=9)$ & $5.92 \pm 0.71(n=19)$ & 0.863 \\
\hline Change of HbAlc (\%) & $\begin{array}{l}-1.10 \pm 1.15 \\
\quad(n=25)\end{array}$ & $\begin{array}{l}-1.47 \pm 1.17 \\
(n=9)\end{array}$ & $\begin{array}{c}-0.90 \pm 1.13 \\
(n=19)\end{array}$ & 0.202 \\
\hline $\begin{array}{l}\text { Pre-pregnancy insulin dose } \\
\quad(\mathrm{U} / \mathrm{kg} \cdot \mathrm{d})\end{array}$ & $0.64 \pm 0.23(n=35)$ & $0.59 \pm 0.24(n=10)$ & $0.66 \pm 0.23(n=25)$ & 0.559 \\
\hline $\begin{array}{l}\text { Late-pregnancy insulin dose } \\
\quad(\mathrm{U} / \mathrm{kg} \cdot \mathrm{d})\end{array}$ & $1.11 \pm 0.44(n=34)$ & $1.01 \pm 0.42(n=10)$ & $1.15 \pm 0.45(n=24)$ & 0.508 \\
\hline Change of insulin dose $(\mathrm{U} / \mathrm{d})$ & $35.30 \pm 22.60(n=31)$ & $35.9 \pm 22.44(n=10)$ & $35.04 \pm 23.22(n=21)$ & 0.933 \\
\hline Change of weight $(\mathrm{kg})$ & $15.21 \pm 10.38(n=37)$ & $13.45 \pm 5.66(n=10)$ & $15.87 \pm 11.69(n=27)$ & 0.706 \\
\hline
\end{tabular}

The bold values are represents that $p$ value is $<0.05$

Data are shown as mean \pm standard deviation (mean \pm SD) or percentage (\%)

$B M I$ body mass index, $H b A l c$ hemoglobin Alc, CS-II continuous subcutaneous insulin infusion

and the other cardiac malformation with a bright spot in the left ventricle. There were $3 / 38$ cases of shoulder dystocia and $2 / 38$ cases of neonatal hyperbilirubinemia in our cohort (Table 2).

Ten patients were treated with metformin during pregnancy. Three of them started metformin from pre-pregnancy, four from early pregnancy and two from late pregnancy. According to the medical records, clinicians prescribed metformin mostly because satisfactory glucose control was not achieved with a high insulin dose (over 0.8 unit $/ \mathrm{kg}$ ). There were no significant differences in age, duration of diabetes, pre-pregnancy BMI and pre-pregnancy insulin dose between metformin-insulin group and insulin-only group $(p>0.05)$. Three patients in the insulin-only group were complicated by chronic diabetic complications, while none in the metformin-insulin group. One of the three patients experienced preeclampsia and delivered a 1660-g male baby in gestational week 30 . The baby was admitted to the NICU as a premature infant. Another patient delivered an LBW neonate at $37+2$ weeks who also experienced neonate hypoglycemia and was admitted to the NICU. Rates of continuous subcutaneous insulin infusion (CS-II) were comparable in the metformininsulin group and insulin-only group, with $30.0 \%$ and $35.7 \%$, respectively. HbA1c in prepregnancy was higher in the metformin-insulin group $(7.65 \pm 1.24 \%$ vs. $\quad 6.92 \pm 1.32 \%, \quad p=$ 0.033). Although not statistically significant, the decrease of HbA1c in the metformin-insulin group was more obvious $(-1.47 \pm 1.17 \%$ vs. $-0.90 \pm 1.13 \%, p=0.202)$. In the third trimester, the HbA1c levels in the two groups were 
Table 2 Perinatal outcomes of T1D patients and comparison of groups

\begin{tabular}{|c|c|c|c|c|}
\hline Variables & & Metformin-insulin & Insulin only & $p$ value \\
\hline 1-Minute Apgar Score & $9.82 \pm 0.73(n=38)$ & $\begin{array}{l}10.00 \pm 0.00 \\
(n=10)\end{array}$ & $9.75 \pm 0.85(n=28)$ & 0.288 \\
\hline Preterm delivery & $10.5 \%(n=38)$ & $0.0 \%(n=10)$ & $14.3 \%(n=28)$ & 0.556 \\
\hline Caesarean section & $65.8 \%(n=38)$ & $50.0 \%(n=10)$ & $71.4 \%(n=28)$ & 0.263 \\
\hline Preeclampsia/eclampsia & $7.9 \%(n=38)$ & $0.0 \%(n=10)$ & $10.7 \%(n=28)$ & 0.552 \\
\hline Polyhydramnios & $5.3 \%(n=38)$ & $10.0 \%(n=10)$ & $3.6 \%(n=28)$ & 0.462 \\
\hline Premature rupture of membranes & $10.5 \%(n=38)$ & $10.0 \%(n=10)$ & $10.7 \%(n=28)$ & 1.000 \\
\hline $\begin{array}{l}\text { Laceration of perineum or lateral } \\
\text { episiotomy }\end{array}$ & $76.9 \%(n=13)$ & $100 \%(n=5)$ & $62.5 \%(n=8)$ & 0.231 \\
\hline $\begin{array}{l}\text { Weight retention in postpartum of } \\
6 \text { weeks }\end{array}$ & $87.1 \%(n=31)$ & $88.9 \%(n=9)$ & $86.4 \%(n=22)$ & 1.000 \\
\hline $\begin{array}{l}\text { Weight retention in postpartum of } \\
6 \text { weeks }(\mathrm{kg})\end{array}$ & $4.85 \pm 4.07(n=26)$ & $4.39 \pm 2.22(n=8)$ & $5.06 \pm 4.71(n=18)$ & 0.781 \\
\hline Birth height $(\mathrm{cm})$ & $\begin{array}{c}49.08 \pm 2.59 \\
(n=37)\end{array}$ & $\begin{array}{l}49.90 \pm 2.47 \\
(n=10)\end{array}$ & $48.78 \pm 2.60(n=27)$ & 0.323 \\
\hline Birth weight (g) & $\begin{array}{l}3513.95 \pm 806.37 \\
\quad(n=38)\end{array}$ & $\begin{array}{l}3817.00 \pm 910.74 \\
\quad(n=10)\end{array}$ & $\begin{array}{l}3405.71 \pm 753.93 \\
\quad(n=28)\end{array}$ & 0.371 \\
\hline LBW/SGA & $7.9 \%(n=38)$ & $0.0 \%(n=10)$ & $10.7 \%(n=28)$ & 0.552 \\
\hline Macrosomia/LGA & $42.1 \%(n=38)$ & $50.0 \%(n=10)$ & $39.3 \%(n=28)$ & 0.713 \\
\hline Neonatal hypoglycemia & $28.9 \%(n=38)$ & $10.0 \%(n=10)$ & $35.7 \%(n=28)$ & 0.225 \\
\hline $\mathrm{NICU}$ & $18.4 \%(n=38)$ & $10.0 \%(n=10)$ & $21.4 \%(n=28)$ & 0.650 \\
\hline Shoulder dystocia & $7.9 \%(n=38)$ & $10.0 \%(n=10)$ & $7.1 \%(n=28)$ & 1.000 \\
\hline Neonatal malformation & $10.5 \%(n=38)$ & $20.0 \%(n=10)$ & $7.1 \%(n=28)$ & 0.279 \\
\hline Neonatal hyperbilirubinemia & $5.3 \%(n=38)$ & $20.0 \%(n=10)$ & $0.0 \%(n=28)$ & 0.064 \\
\hline
\end{tabular}

Data are shown as mean \pm standard deviation (mean \pm SD) or percentage (\%)

$B M I$ body mass index, NICU neonatal intensive care unit, $L B W$ low birth weight, $S G A$ small for gestational age, $L G A$ large for gestational age

equivalent $\quad(6.09 \pm 1.05 \%$ vs. $5.92 \pm 0.71 \%$, $p=0.863)$. One-way ANOVA did not indicate that the levels of baseline HbA1c had an impact on the levels of HbA1c reduction (data not shown). Whether in pre-pregnancy or in the third trimester, there was no difference in the amount of insulin between the two groups. Patients in the metformin-insulin group gained less weight during pregnancy; however, no statistical difference was reached (Table 1). In all, there was no difference in perinatal outcomes between the two groups. When patients with chronic diabetic complications were excluded, there was still no difference in perinatal events (date not showed). However, the metformin-insulin group seemed to experience a lower incidence rate than the insulin-only group in most adverse perinatal outcomes we investigated (Table 2). 


\section{DISCUSSION}

It is universally recognized that insulin resistance plays a vital role in pregnancy, as the placenta physically releases progesterone, prolactin and cortisol. Insulin resistance has also been proposed as a critical reason for suboptimal glycemic control in T1D [10], although the traditional view is still that T1D results from an autoimmune destruction of pancreatic $\beta$-cells, and the predominant pathophysiology is an essentially absolute insulin deficiency. Insulin resistance is associated with increased insulin dose requirements and further weight gain. Including 237 pregnancies affected by T1D, a study indicated that the daily insulin dose increased by 52 units per day on average [11]. Consistent with the previous study, in our study, the amount of insulin in pregnancy increased $33.72 \pm 23.38$ units/day on average. Similar to other studies, obesity was more prevalent in T1D women than in non-diabetic women. In our study, $35.1 \%$ patients were overweight before pregnancy, higher than the reported non-diabetic overweight incidence (14.4\%) [12]. It is reported that maternal BMI and gestational weight gain are critical risk factors for poor perinatal outcomes [13-15]. Women who seemingly achieve adequate glycemic control in pregnancy continue to experience a higher risk of excess fetal growth, leading to LGA or macrosomia [16]. In our study, the mean $\mathrm{HbA} 1 \mathrm{c}$ was $5.98 \pm 0.82 \%$ in the third trimester. However, 16 neonates (42.1\%) had macrosomia/LGA, which was the most common neonatal complication. We found that gestational weight gain might explain the high incidence rate of macrosomia/ LGA.

There has been growing interest in exploring the role of metformin as an adjunct to insulin therapy in T1D, with the aim of improving insulin sensitivity and glycemic control, limiting the insulin dose and weight gain. REMOVAL was the largest multicenter, double-blind, placebo-controlled trial assessing the effect of metformin over 3 years in 428 adults (age > 40 years) with T1D. HbA1c was reduced by metformin over 3 years $(-0.13 \%, \quad 95 \%$ CI
-0.22 to $-0.04, p=0.006)$, which was primarily accounted for by a reduction at 3 months $(-0.24 \%, 95 \% \mathrm{CI}-0.34$ to $-0.13, \mathrm{p}<0.0001)$. REMOVAL also demonstrated a favorable effect of metformin on body weight, insulin dose requirement and lipids, similar to previous meta-analysis of smaller studies. However, as metformin can go across the placenta, clinicians are concerned about the safety of metformin during pregnancy. Recently, increasing evidence has supported the safety of metformin during pregnancy [17]. A meta-analysis including five RCTs revealed less weight gain and fewer hypertension disorders in the metformin group [18]. A systematic review published in the British Medical Journal (BMJ) in 2015 found a significant downward trend in the risk of hypoglycemia in the metformin group [19]. Metformin may further reduce NICU admission and cesarean delivery rates [20]. Compared with insulin alone, metformin as adjunct therapy in T2D pregnant women with insulin resistance was valuable; it could reduce hypoglycemia, hospital stay days and respiratory distress syndrome [21]. We retrospectively compared glucose management and perinatal outcomes between pregnant women with T1D on metformin plus insulin and those on insulin only. As we mentioned before, metformin was usually prescribed in cases of poor glucose control with high-dose insulin in our study, resulting in higher baseline HbA1c levels in the metformininsulin group. Our study suggested that although there was a higher HbA1c level in the metformin-insulin group in pre-pregnancy, the HbA1c decreased more pronouncedly in the metformin-insulin group without increasing the insulin dosage. It indicated that adjunct metformin in pregnant women with T1D with poor glycemic control may be valuable. Although no perinatal outcomes achieved statistical significance, maternal weight gain, neonatal hypoglycemia and NICU admission, neonatal hypoglycemia and NICU admission presented a declining trend, which is consistent with studies of pregnant women with T2D [21]. Since three patients in the insulin-alone group had chronic diabetic complications and two of them experienced adverse perinatal outcomes, which might bias the results, we likewise carried out statistical 
analysis excluding these three patients, and the results corresponded with the previous ones. The metformin-insulin group seemed to experience more neonatal malformation, neonatal hyperbilirubinemia and macrosomia, but no statistically significant differences were reached. In summary, we discovered no increased adverse perinatal events with metformin therapy.

This pioneering study fills in the knowledge gap concerning metformin in T1D patients during pregnancy, providing a justification for further prospective and large-scale studies. Nevertheless, there are some limitations to our study. First, T1D prevalence is much lower in China than in Caucasian populations [22]. Moreover, a nationwide cohort study in Taiwan recently reported that T1D greatly impairs female fertility [23]. As a result, although all pregnant women with T1D who gave birth between 1 January 2006 and 31 May 2018 were included, the sample size of the study was relatively small. The statistical power was insufficient for limited subject numbers, especially in evaluating the perinatal outcomes. It is appropriate to consider this as a pioneering study, and definite conclusions should be drawn with caution. Second, levels of pre-pregnancy HbA1c were higher in the metformin-insulin group. Generally, the higher the baseline $\mathrm{HbA1c}$, the greater the HbA1c reduction that might be seen after intervention; that the patients in this group might be more motivated could be one of the reasons. Further prospective studies are needed in patients with equivalent baseline HbA1c levels. Third, as a retrospective study, it was impractical to thoroughly investigate the adverse drug reactions to metformin, such as gastrointestinal discomfort. Fourth, previous studies indicated that metformin helps to reduce oxidative stress and may have a direct beneficial effect on insulin secretion impaired by oxidative stress in animal models and non-pregnant diabetic patients [24, 25]; we propose that the benefits of metformin beyond glycemic control itself makes it a promising adjunct treatment in pregnant type 1 diabetes patients. However, as a retrospective study, we were unable to measure cytokines in these patients.

\section{CONCLUSIONS}

It is vital to explore whether oral anti-diabetic medication has its place in pregnant women with T1D, as insulin resistance plays an important role and glucose management was usually unsatisfactory for patients solely on insulin. This research fills the knowledge gap in the field of metformin use in pregnant women with T1D, suggesting that metformin might be safe and effective in these patients. Larger and prospective studies are needed to confirm this.

\section{ACKNOWLEDGEMENTS}

We thank the participants of the study.

Funding. This work was supported by grants from the National Key R\&D Program of China (2017YFC1309603), National Key Research and Development Program of China (2016YFA 0101002), National Natural Science Foundation of China (nos. 81170736, 81570715, 81870579), Medical Epigenetics Research Center and Chinese Academy of Medical Sciences (2017PT 31036, 2018PT31021). However, the study sponsor did not fund the journal's article processing charges; these were funded by the authors.

Authorship. All named authors meet the International Committee of Medical Journal Editors (ICMJE) criteria for authorship for this article, take responsibility for the integrity of the work as a whole and have given their approval for this version to be published.

Disclosures. Fan Ping: a physician at the Department of Endocrinology in $\mathrm{PUMCH}$, who focuses on the management of diabetic pregnancy. Mingqun Deng: postgraduate student at PUMCH. Xiao Zhai: postgraduate student at PUMCH. Yingna Song: an obstetrician in the Department of Gynaecology and Obstetrics at PUMCH. Xinhua Xiao: a professor in the Department of Endocrinology at PUMCH; he has focused on investigating the role of the intrauterine environment in glucose 
metabolism for many years. The authors have no other relevant disclosures.

Compliance with Ethics Guidelines. This retrospective study was approved by the Ethics Committee of PUMCH (approval no. ZS-1380), and written informed consent was obtained from all patients. All procedures performed in studies involving human participants were in accordance with the ethical standards of the Ethics Committee of PUMCH and with the 1964 Helsinki Declaration and its later amendments or comparable ethical standards.

Data Availability. The datasets used and/or analyzed during the current study are available from the corresponding author on reasonable request.

Open Access. This article is distributed under the terms of the Creative Commons Attribution-NonCommercial 4.0 International License (http://creativecommons.org/licenses/ by-nc/4.0/), which permits any noncommercial use, distribution, and reproduction in any medium, provided you give appropriate credit to the original author(s) and the source, provide a link to the Creative Commons license, and indicate if changes were made.

\section{REFERENCES}

1. Maresh MJ, Holmes VA, Patterson CC, et al. Glycemic targets in the second and third trimester of pregnancy for women with type 1 diabetes. Diabetes Care. 2015;38(1):34-42.

2. Wahabi HA, Alzeidan RA, Esmaeil SA. Pre-pregnancy care for women with pre-gestational diabetes mellitus: a systematic review and meta-analysis. BMC Public Health. 2012;12:792.

3. Hillman N, Herranz L, Vaquero PM, Villarroel A, Fernandez A, Pallardo LF. Is pregnancy outcome worse in type 2 than in type 1 diabetic women? Diabetes Care. 2006;29(11):2557-8.

4. Persson M, Norman M, Hanson U. Obstetric and perinatal outcomes in type 1 diabetic pregnancies: a large, population-based study. Diabetes Care. 2009;32(11):2005-9.
5. Murphy HR, Bell R, Cartwright C, et al. Improved pregnancy outcomes in women with type 1 and type 2 diabetes but substantial clinic-to-clinic variations: a prospective nationwide study. Diabetologia. 2017;60(9):1668-777.

6. Garcia-Patterson A, Gich I, Amini SB, Catalano PM, de Leiva A, Corcoy $\mathrm{R}$. Insulin requirements throughout pregnancy in women with type 1 diabetes mellitus: three changes of direction. Diabetologia. 2010;53(3):446-51.

7. Cyganek K, Klupa T, Szopa M, Katra B, Malecki MT. Medical care of pregnant women with type 1 diabetes: current guidelines and clinical practice. Pol Arch Med Wewn. 2013;123(1-2):59-655.

8. Moon RJ, Bascombe LA, Holt RI. The addition of metformin in type 1 diabetes improves insulin sensitivity, diabetic control, body composition and patient well-being. Diabetes Obes Metab. 2007;9(1):143-5.

9. Gray SG, McGuire TM, Cohen N, Little PJ. The emerging role of metformin in gestational diabetes mellitus. Diabetes Obes Metab. 2017;19(6):765-72.

10. Kirti K, Maria A, Michael R. Insulin resistance in type 1 diabetes mellitus. Metabolism. 2015;64(12):1629-39.

11. Steel JM, Johnstone FD, Hume R, Mao JH. Insulin requirements during pregnancy in women with type I diabetes. Obstet Gynecol. 1994;83(2):253-8.

12. Xi B, Liang $\mathrm{Y}, \mathrm{He} \mathrm{T}$, et al. Secular trends in the prevalence of general and abdominal obesity among Chinese adults, 1993-2009. Obes Rev. 2012;13(3):287-96.

13. Abell SK, Boyle JA, de Courten B, et al. Contemporary type 1 diabetes pregnancy outcomes: impact of obesity and glycaemic control. Med J Aust. 2016;205(4):162-7.

14. Evers IM, de Valk HW, Mol BW, ter Braak EW, Visser GH. Macrosomia despite good glycaemic control in Type I diabetic pregnancy; results of a nationwide study in The Netherlands. Diabetologia. 2002;45(11):1484-9.

15. Goldstein RF, Abell SK, Ranasinha S, et al. Association of gestational weight gain with maternal and infant outcomes: a systematic review and metaanalysis. JAMA. 2017;317(21):2207-25.

16. McGrath RT, Glastras SJ, Hocking SL, Fulcher GR. Large-for-gestational-age neonates in type 1 diabetes and pregnancy: contribution of factors beyond hyperglycemia. Diabetes Care. 2018;41(8):1821-8. 
17. Cassina M, Dona M, Di Gianantonio E, Litta P, Clementi M. First-trimester exposure to metformin and risk of birth defects: a systematic review and meta-analysis. Hum Reprod Update. 2014;20(5):656-69.

18. Gui J, Liu Q, Feng L. Metformin vs insulin in the management of gestational diabetes: a meta-analysis. PLoS One. 2013;8(5):e64585.

19. Balsells M, Garcia-Patterson A, Sola I, Roque M, Gich I, Corcoy R. Glibenclamide, metformin, and insulin for the treatment of gestational diabetes: a systematic review and meta-analysis. BMJ. 2015;350:h102.

20. Kitwitee P, Limwattananon S, Limwattananon C, et al. Metformin for the treatment of gestational diabetes: an updated meta-analysis. Diabetes Res Clin Pract. 2015;109(3):521-32.

21. Ibrahim MI, Hamdy A, Shafik A, Taha S, Anwar M, Faris $M$. The role of adding metformin in insulinresistant diabetic pregnant women: a randomized controlled trial. Arch Gynecol Obstet. 2014;289(5):959-65.

22. Weng J, Zhou Z, Guo L, et al. Incidence of type 1 diabetes in China, 2010-13: population based study. Bmj. 2018;360:j5295.

23. Lin YH, Chen KJ, Peng YS, Chen PC, Yang YH. Type 1 diabetes impairs female fertility even before it is diagnosed. Diabetes Res Clin Pract. 2018;143:151-8.

24. Singh RK, Gupta B, Tripathi K, Singh SK. Anti oxidant potential of Metformin and Pioglitazone in Type 2 Diabetes Mellitus: Beyond their anti glycemic effect. Diabetes Metab Syndr Clin Res Rev. 2016;10(2):102-4.

25. Piro S, Rabuazzo AM, Renis M, Purrello F. Effects of metformin on oxidative stress, adenine nucleotides balance, and glucose-induced insulin release impaired by chronic free fatty acids exposure in rat pancreatic islets. J Endocrinol Invest. 2012;35(5):504-10. 